\title{
A promising approach to targeting type 1 IFN in systemic lupus erythematosus
}

\author{
Yashaar Chaichian, ${ }^{1}$ Daniel J. Wallace, ${ }^{2}$ and Michael H. Weisman ${ }^{2}$ \\ 'Division of Immunology and Rheumatology, Stanford University, Palo Alto, California, USA. 'Division of Rheumatology, Cedars-Sinai Medical Center, David Geffen School of Medicine, \\ UCLA, Los Angeles, California, USA.
}

Despite advances in understanding systemic lupus erythematosus (SLE) pathogenesis, most clinical trials of new targeted therapies have been met with disappointment. The type I IFN pathway is believed to play an important role in SLE, and the proposed involvement of this pathway helps explain the frustration behind the failure at targeting either IFN- $\alpha$ or the type 1 IFN receptor itself. In this issue of the $J C I$, Furie et al. report on an intriguing phase $1 \mathrm{~b}$ study that demonstrates an approach for inhibiting this pathway in the skin using an $\mathrm{mAB}$ (BIIB059) that targets the blood DC antigen 2 (BDCA-2) receptor on plasmacytoid DCs (pDCs). BIIB059 decreased IFN expression and improved cutaneous lupus disease activity, with a favorable safety profile. Whether or not this strategy will be effective in managing SLE in other organs remains unanswered. However, these results suggest that closing the door on targeting the type 1 IFN pathway in SLE may be premature and highlight the emerging question of whether an organ-specific approach toward lupus trials and treatment should be the wave of the future.

\section{SLE treatment - unmet need and limitations of previous clinical trials}

Disease remission remains an elusive goal for many patients with systemic lupus erythematosus (SLE) (1), even as mortality rates for SLE have significantly improved over time (2). Furthermore, even among those who do respond adequately to the current standard-of-care management, these medications are nonspecific in their immunosuppressive approach and their toxicity profile is often unacceptable for a chronic illness such as SLE. Health-related quality of life measures remain low among many SLE patients (3). There is still a large unmet need for optimization of lupus management.
The advent of biologic therapies has dramatically changed the landscape for en diseases, notably rheumatoid arthritis, psoriasis, multiple sclerosis, and inflammatory bowel disease, over the past 20 years. Unfortunately, the same cannot be said about SLE, as nearly all novel therapies that target specific pathways of immune dysregulation in lupus have met with failure. Amidst this backdrop exist signs of hope. Phase II study results of baricitinib, an oral selective and reversible JAK1 and JAK2 inhibitor (4), and ustekinumab, an anti-IL-12/23 mAb (5), have been promising. Post hoc analysis from a phase II study (6) of the anti-IL-6 mAB PF-04236921 managing several other autoimmune-driv-

\section{Related Article: p. 1359}

Conflict of interest: YC has received support from Gilead Sciences and AMPEL BioSolutions to conduct clinical trials and from Pfizer to help facilitate a patient-centered care program. He has received support from GSK to attend an advisory board meeting. DJW has consulted for CSK, Merck, EMD Serono, Lilly, and Amgen. MHW has consulted for Takeda, Lilly, SetPoint Medical, AbbVie, and Boehringer Ingelheim and has received legal fees from Tharpe \& Howell LLP as well as Paul Hastings.

Reference information: / Clin Invest. 2019;129(3):958-961. https://doi.org/10.1172/JCI127101. revealed efficacy and safety, and trends toward treatment response were observed in a recent phase IIb trial (7) and long-term extension study (8) of atacicept, a dual inhibitor of a proliferation-inducing ligand (APRIL), and B cell activating factor of the TNF family (BAFF). Nevertheless, belimumab (anti-BAFF $\mathrm{mAb}$ ) remains the only FDA-approved therapy for SLE in the past 60 years (9), and many clinicians regard the impact of this drug as modest at best.

Multiple reasons have been cited for the lack of successful clinical trials in SLE (10). Disease heterogeneity can make it difficult to identify the specific patient phenotype for enrollment in a trial. Appropriate criticism has been levied against the general approach toward background medications in lupus clinical trials, as it is difficult to achieve primary end points and demonstrate a clinically meaningful response compared with standard of care when the target groups are on multiple immunosuppressive drugs plus glucocorticoids. The use of rescue therapies for disease flares can also impede the ability to detect a beneficial effect of study drug. Another concern has been the lack of a consistent outcome measure that is clinically meaningful, easy to employ, and derived from clinical trial data, as opposed to either expert opinion (SLE Disease Activity Index [SLEDAI]) or observational cohorts (British Isles Lupus Assessment Group [BILAG]) (11). The recruitment challenges for SLE trials are readily acknowledged; however, some studies have been too small and thus make it more difficult to detect a statistically significant difference between treatment and placebo arms. Even when there have been positive results in the overall study population for a specific treatment, the results have often been underpowered to assess whether one subgroup of patients may benefit more than another. Finally, parallel phase III trials of the same study drug present a challenge, as they do not allow lessons learned from the failure of one trial to be applied to the other. 


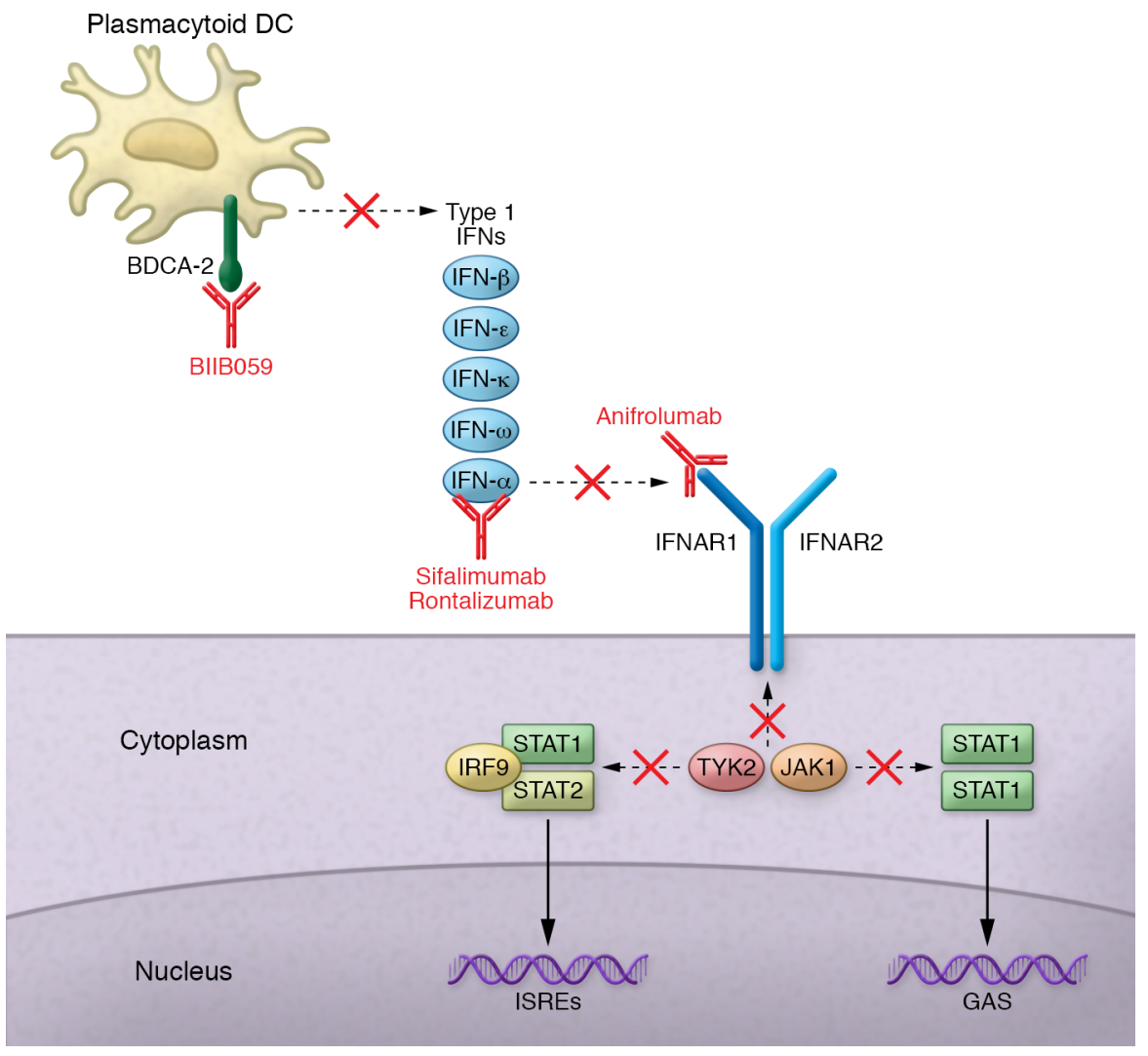

Figure 1. Sites of action of drugs that inhibit type 1 IFN. In this issue, Furie et al. demonstrate that BIIB059 is an mAb that works upstream of other type 1 IFN-interfering drugs. BIIB059 binds the BDCA-2 receptor on pDCs, leading to inhibition of type 1 IFN production. Sifalimumab and rontalizumab act as mAbs against IFN- $\alpha$, blocking all and most IFN- $\alpha$ subtypes, respectively. Anifrolumab is an mAb against IFNAR, preventing interaction with tyrosine kinase 2 (TYK2) and JAK1 and thus inhibiting the ability of IFN- $\alpha$ and all other type 1 IFNs to mediate downstream effects. IRF9, IFN regulatory factor 9; ISRE, IFN-stimulated response element; GAS, IFN- $\gamma$-activated sequence.

Type 1 IFN in SLE: immunopathogenesis, deferred clinical breakthroughs

While these explanations are all valid, the exact reasons why SLE clinical trials to date have been underwhelming may also derive from the complexity of lupus immunopathogenesis, which could even evolve over time in a single patient, and the need to identify suitable organ-specific therapeutic targets. For a number of years, the focus of development of new targeted therapies in SLE was directed primarily toward perturbation or ablation of $\mathrm{T}$ cells and B cells. However, we know that innate immunity also plays a critical role in SLE pathogenesis, with defects in antigen-presenting cells, TLRs, and cytokine pathways all having been implicated in disease (12). The intricate interplay of the immune system means that defects in innate immunity directly affect $\mathrm{T}$ cell and
B cell activity; counteracting this particular dysregulation in SLE may have profound therapeutic implications.

Type 1 IFN is one innate immune pathway that is aberrant in many lupus patients. Activation of TLR7 and TLR9 is thought to be central to induction of a type 1 IFN response, although TLR7/9 inhibition studies have been unsuccessful. Downstream effects of increased type 1 IFN expression are variable and include an increase in neutrophil extracellular traps (NETs) from activated neutrophils, B cell hyperreactivity via increased BAFF production, and loss of Treg function (12). Mouse models of SLE have shown that IFN is pathogenic; exogenous IFN- $\alpha$ leads to worsening disease (13). Results from prior SLE clinical trials directly targeting the type 1 IFN pathway have been decidedly mixed, but nevertheless, these studies implicate a pathogenic role of this pathway in humans $(14,15)$.
Given the perceived biologic complexities of the role type 1 IFN plays in lupus pathogenesis, the disappointment that has followed contemporaneous clinical trials targeting this pathway has been understandable. mAbs directed against IFN- $\alpha$ were initially studied; however, rontalizumab failed to meet its primary and secondary end points (16), and sifalimumab had underwhelming efficacy despite meeting its primary and some secondary end points (14). Furthermore, the specificity toward IFN- $\alpha$ meant that other type 1 IFNs were not blocked and were free to bind the type 1 IFN receptor (IFNAR) and mediate cell signaling. As

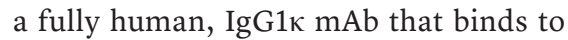
IFNAR, anifrolumab has the advantage of inhibiting signaling by all type I IFNs. Phase II results in patients with moderate-to-severe lupus were quite promising (15), with a clinically significant response in subjects with high baseline IFN gene expression, which makes biologic sense, and a low rate of adverse events (AEs) overall. Unfortunately, the recent phase III TULIP 1 study of anifrolumab failed to meet the primary end point (17). Furthermore, the clinically important patient-reported outcomes (PRO), which may reflect how patients actually feel when taking the drug, did not significantly improve in any of these studies (D.J. Wallace, personal communication). Based on the results from these trials, it appears that further investigation into targeting IFN- $\alpha$ or IFNAR directly will not go forward.

\section{A new target and an organ-specific approach in IFN-directed therapy?}

In contrast to the above referenced studies, a unique means of inhibiting the production of type 1 IFN at the level of the plasmacytoid DCs (pDCs) (Figure 1) was undertaken by Furie et al. (18) and reported in this issue. We know that pDCs are the primary cell source of the type 1 IFN signal in SLE. Blood DC antigen 2 (BDCA-2) is a pDC-specific receptor that blocks type 1 IFN production when bound. Blomberg et al. (19) previously reported that SLE patients not only have decreased numbers of BDCA-2-expressing $\mathrm{pDCs}$, but that IFN- $\alpha$ production could be mitigated using an anti-BDCA- 
2/4 mAb. Subsequently, Wu et al. (20) demonstrated that decreased expression of BDCA-2 on activated pDCs in SLE is driven by TLR7 or TLR9 agonists.

These and similar mechanistic studies cited in the paper help inform the rationale for the development of a humanized BDCA-2-binding mAb (BIIB059). Furie et al. conducted a multicenter, randomized, double-blind, placebo-controlled phase 1b study (18) assessing the safety, tolerability, pharmacokinetic, and pharmacodynamic effects of BIIB059 in healthy volunteers and patients with SLE with active cutaneous disease. The authors also evaluated the biological activity and clinical response among these cutaneous lupus patients. As such, this was an organ-specific study essentially limited to SLE patients with active skin involvement. The study was divided into three parts: single ascending doses of BIIB059 in healthy volunteers (part 1), a single dose (20 mg/ $\mathrm{kg}$ i.v.) among SLE patients (part 2), and multiple ascending doses in both healthy volunteers and SLE patients (part 3). The report in this issue focuses on the findings of parts 1 and 2. Patients with SLE $(n=12)$ met 1997 American College of Rheumatology (ACR) criteria and were required to have active skin disease, though no specific baseline cut-off regarding cutaneous disease activity measure (Cutaneous Lupus Erythematosus Disease Area and Severity Index Activity [CLASI-A] score) was required. Similarly, there was no requirement in terms of baseline SLEDAI-2K activity, a systemic disease activity measure. After a 3-day treatment and outpatient observation period, patients in parts 1 and 2 were followed up for 16 weeks, with a further 12-week follow-up for part 2 to monitor AEs.

Furie and colleagues confirmed that BIIB059 resulted in BDCA-2 internalization on pDCs, a necessary step for facilitating inhibition of type 1 IFN. Administration of BIIB059 also reduced expression of IFN response genes in whole blood and reduced expression of IFN response proteins within affected skin sample biopsies. The effect on the IFN signature in the skin correlated with reductions in cutaneous disease, as measured by the CLASI-A score. Together, these results provide strong evidence of the biological activity of BIIBO59 in SLE patients with active cutaneous involvement. Moreover, single doses of BIIB059 had favorable pharmacokinetic and pharmacodynamic profiles. Importantly, BIIB059, as administered in this study, was generally safe and well tolerated. Most AEs observed were mild to moderate in severity.

\section{Conclusions and future directions}

The exciting results from Furie et al. suggest that the decision to continue investigating the potential benefits of targeting a novel vantage point within the important type 1 IFN pathway was astute. These findings also raise a number of intriguing questions to consider. This was a small study administering BIIB059 in a limited fashion with a short follow-up interval, all of which are appropriate, considering this was a phase I trial. As the number of patients with extracutaneous lupus disease involvement was quite low, it is not possible to extrapolate the impact of BIIB059 on patients with these sites of disease involvement. It is hoped that the planned phase II study with a longer duration of treatment will provide necessary additional information regarding the efficacy of this agent in a larger, more phenotypically diverse cohort of SLE patients. Additional safety data will be essential, as prior IFN-targeted therapies demonstrated a herpes zoster signal. In the Furie et al. trial, one BIIB059-treated patient developed a herpes zoster infection that was felt to be related to the study drug, but was not considered serious in severity.

Furie and colleagues have clearly demonstrated proof of biological activity of BIBB059 in SLE patients, given the reductions in IFN expression in both whole blood and lesional skin. However, due to the small sample size, firm conclusions cannot be drawn regarding whether responsiveness to this drug differs between SLE patients with low versus high baseline levels of IFN gene expression. One would hypothesize that patients with higher IFN levels would be more responsive; demonstrating this personalized medicine approach could potentially allow the selection of a suitable SLE patient population in advance. Only 2 out of $8 \mathrm{SLE}$ patients that received BIIB059 were on antimalarial therapy, which inhibits TLR7/9 as its principal mechanism of action. As both medications interfere with the type 1 IFN pathway, additional data on response rates in a larger cohort of patients receiving BIIB059 plus an antimalarial will be instructive.

Given the potential importance of IFN in SLE pathogenesis, it is reasonable to assume that the skin may not be the only site of disease activity that would respond to BIIB059. As the complexity of SLE pathogenesis is well known, what works in one target organ may not in other affected organs. The observation that some patients with cutaneous lupus have minimal or absent signs of systemic disease activity opens up the possibility that an organ-specific treatment approach unique to the skin may be preferential. Nevertheless, it is worth pondering the following: which sites of noncutaneous SLE organ involvement will most benefit from BIIB059? Will the use of other validated measures to assess global disease activity be able to demonstrate a clinically and statistically significant treatment effect with this study drug? Or will we need to focus on organ-specific measures of disease activity in assessing the efficacy of BIIB059 at other disease sites? Once we identify which organ or organs will respond best to this treatment approach, how does this advance our knowledge of potential immune mechanisms in SLE?

It is important to remember that SLE is a chronic illness with ups and downs over a long period of time. Ultimately, additional studies determining the organ specificity (or lack thereof) of targeting the type 1 IFN pathway at the level of the pDC via BIIB059 will provide, not only answers as to the precise role of this treatment approach in SLE, but it is hoped, additional insights into disease immunopathogenesis as well. For a disease with such a large unmet need, this would be welcome news indeed.

\section{Acknowledgments}

We would like to acknowledge Mark Genovese for his helpful input on this Commentary.

Address correspondence to: Yashaar Chaichian, 1000 Welch Rd., Suite 203, MC 5755, Division of Immunology and Rheumatology, Stanford University, Palo Alto, California 94304, USA. Phone: 650.725.8004; Email:ychaich@stanford.edu. 
1. Wilhelm TR, Magder LS, Petri M. Remission in systemic lupus erythematosus: durable remission is rare. Ann Rheum Dis. 2017;76(3):547-553.

2. Singh RR, Yen EY. SLE mortality remains disproportionately high, despite improvements over the last decade. Lupus. 2018;27(10):1577-1581.

3. Yazdany J, Yelin E. Health-related quality of life and employment among persons with systemic lupus erythematosus. Rheum Dis Clin North Am. 2010;36(1):15-32, vii.

4. Wallace DJ, et al. Baricitinib for systemic lupus erythematosus: a double-blind, randomised, placebo-controlled, phase 2 trial. Lancet. 2018;392(10143):222-231.

5. van Vollenhoven RF, et al. Efficacy and safety of ustekinumab, an IL-12 and IL-23 inhibitor, in patients with active systemic lupus erythematosus: results of a multicentre, double-blind, phase 2, randomised, controlled study. Lancet. 2018;392(10155):1330-1339.

6. Wallace DJ, et al. Efficacy and safety of an interleukin 6 monoclonal antibody for the treatment of systemic lupus erythematosus: a phase II dose-ranging randomised controlled trial. Ann Rheum Dis. 2017;76(3):534-542.

7. Merrill JT, et al. Efficacy and safety of atacicept in patients with systemic lupus erythematosus: results of a twenty-four-week, multicenter, randomized, double-blind, placebo-controlled, parallel-arm, phase IIb study. Arthritis Rheumatol.
2018;70(2):266-276.

8. Morand E, et al. OP0251 Attainment of low disease activity and remission in systemic lupus erythematosus patients with high disease activity in the atacicept phase iib address ii study and its long-term extension. Ann Rheum Dis. 2018;77:174-175.

9. Horowitz DL, Furie R. Belimumab is approved by the FDA: what more do we need to know to optimize decision making? Curr Rheumatol Rep. 2012;14(4):318-323.

10. Wallace DJ. Improved strategies for designing lupus trials with targeted therapies: learning from 65 years of experience. Lupus. 2016;25(10):1141-1149.

11. Forbess LJ, Bresee C, Wallace DJ, Weisman $\mathrm{MH}$. Failure of a systemic lupus erythematosus response index developed from clinical trial data: lessons examined and learned. Lupus. 2017;26(9):909-916.

12. Tsokos GC, Lo MS, Costa Reis P, Sullivan KE. New insights into the immunopathogenesis of systemic lupus erythematosus. Nat Rev Rheumatol. 2016;12(12):716-730.

13. Liu Z, Davidson A. IFN $\alpha$ inducible models of murine SLE. Front Immunol. 2013;4:306.

14. Khamashta M, et al. Sifalimumab, an antiinterferon- $\alpha$ monoclonal antibody, in moderate to severe systemic lupus erythematosus: a randomised, double-blind, placebo-controlled study. Ann Rheum Dis. 2016;75(11):1909-1916.

15. Furie R, et al. Anifrolumab, an anti-interferon- $\alpha$ receptor monoclonal antibody, in moderate-to-severe systemic lupus erythematosus. Arthritis Rheumatol. 2017;69(2):376-386.

16. Kalunian KC, et al. A Phase II study of the efficacy and safety of rontalizumab (rhuMAb interferon- $\alpha$ ) in patients with systemic lupus erythematosus (ROSE). Ann Rheum Dis. 2016;75(1):196-202.

17. Lupus Research Alliance. Lupus Research Alliance: Disappointed but Undaunted by Anifrolumab Results. LRA website. https://www.lupusresearch. org/lupus-research-alliance-disappointedundaunted-anifrolumab-results/. Published September 4, 2018. Accessed February 3, 2019.

18. Furie R, et al. Monoclonal antibody targeting BDCA2 ameliorates skin lesions in systemic lupus erythematosus. J Clin Invest. 2019;129(3):1359-1371.

19. Blomberg S, Eloranta ML, Magnusson M, Alm GV, Rönnblom L. Expression of the markers BDCA- 2 and BDCA- 4 and production of interferon-alpha by plasmacytoid dendritic cells in systemic lupus erythematosus. Arthritis Rheum. 2003;48(9):2524-2532.

20. Wu P, et al. TLR9/TLR7-triggered downregulation of BDCA2 expression on human plasmacytoid dendritic cells from healthy individuals and lupus patients. Clin Immunol. 2008;129(1):40-48. 